\title{
Cellular 4G LTE Aeronautical Mobile Telemetry Flight Test Results
}

\author{
Eric Beck ${ }^{1}$, Shobha Erramilli', Sarry Habiby ${ }^{1}$, William Johnson ${ }^{1}$, Achilles Kogiantis ${ }^{1}$, Nan Maung ${ }^{1}$, \\ Kiran Rege ${ }^{1}$, Anthony Triolo ${ }^{1}$, Jeffrey Young ${ }^{1}$ \\ ${ }^{1}$ Perspecta Labs, 331 Newman Springs Road, Red Bank, New Jersey 07701
}

\begin{abstract}
:
Flight test results on a novel approach to Aeronautical Mobile Telemetry (AMT) employing 4G Long Term Evolution (LTE) Cellular Technology are presented. In this system, Doppler shifts are handled by an appliqué providing a front-end to a Commercial-Off-The-Shelf (COTS) LTE modem. The appliqué performs Doppler compensation via real time analysis of LTE signals and allows seamless operation of LTE signaling, including connection setup and handover events. The flight test results clearly demonstrate the capability of the system to sustain an uninterrupted LTE connection at varying aircraft speeds that produce Doppler shifts that are significantly higher than what typical commercial LTE systems can handle. In these tests, the radio link delivered substantial bi-directional data rates up to a maximum range of $60 \mathrm{~km}$, while the combination of Doppler compensation and handovers at the TA provided an uninterrupted radio link in both directions. Overall, the flight test confirmed that an airborne COTS LTE transceiver in combination with a Doppler compensation appliqué can successfully operate at high aircraft speeds. This proof-of-concept flight test also forms a basis from which to further refine AMT solutions based on $4 \mathrm{G}$, and the emerging $5 \mathrm{G}$ technologies.
\end{abstract}

Key words: "Test Range," Telemetry, Aeronautical, Mobile, Cellular, LTE, 4G, Handover, Speed, "Doppler Shift," "Frequency Offset," Compensation, "Flight Test".

\section{Introduction}

Aeronautical Mobile Telemetry (AMT) for test ranges is typically implemented with point-topoint radio links using a wide-beam antenna on the Test Article (TA) that is paired with a highgain parabolic ground station antenna pointing to and tracking the TA. The spectrum used by the radio link is dedicated to that link for the duration of the test. As pointed out in [1] and [2], an alternative implementation of AMT, using a cellular network employing $4^{\text {th }}$ generation 3GPP LTE standards [3], offers several potential benefits over current practice. However, in order to implement such a system, a few technical hurdles need to be overcome. The most formidable of these technical challenges is one posed by the high Doppler shifts in the uplink (UL) signals encountered in an AMT system. In the 4G LTE-based AMT system described in [2], the problem of high Doppler shifts in the UL signal is overcome by deploying a Doppler compensation appliqué between the LTE terminal (located in the TA) and its transmit antennas. (See fig. 1 below.) The appliqué estimates the Doppler shift by processing the UL signals transmitted by the LTE terminal and proactively compensates for the shift expected to be present in the signals as they arrive at the base station receiver. As a result, UL signals arriving at the base station have their center frequency close to where the base station receiver expects it to be.

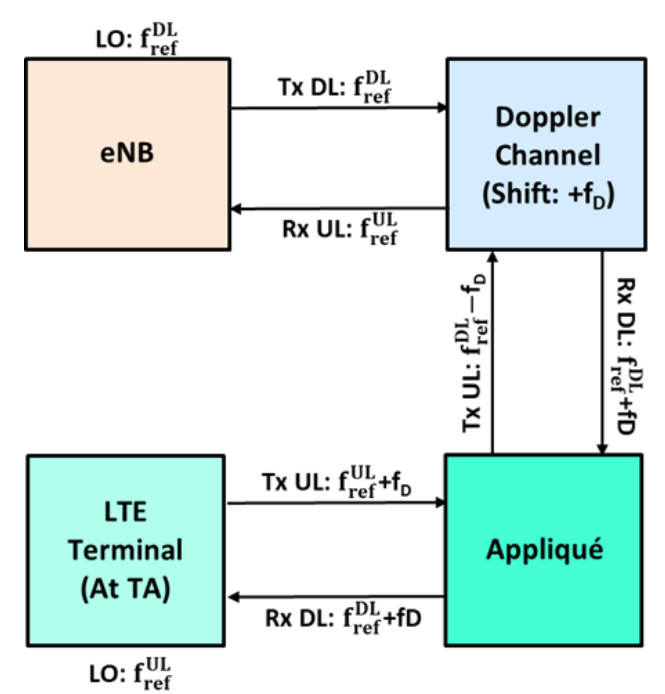

Fig. 1. A Schematic of LTE-Based System with a Doppler Compensation Appliqué

The combination of a COTS LTE terminal and the appliqué has been extensively tested in the lab with a variety of simulated environments. As 
the next step in proving the viability of the LTEbased AMT system, a set of flight tests was planned and executed at Edwards Air Force Base (EAFB) in October, 2019. This paper presents the test setup that was used in the flight tests and describes the highlights of the results of these tests. Over all, the flight tests provided positive confirmation of the viability of an LTEbased AMT system. They also brought out the key features of system behavior that point out the areas where further refinement may help improve the overall system performance.

The rest of this paper is organized as follows: In Section 2, we describe the test setup at EAFB including the layout of the terrestrial segment of the LTE network. Section 3 presents a discussion of the results obtained in the flight tests and their analysis. Finally, Section 4 rounds out the paper by summarizing our conclusions.

\section{Flight Test Setup}

The proposed AMT system will essentially comprise a complete cellular network based on the 3GPP LTE standard [3], which was developed by the $3^{\text {rd }}$ Generation Partnership Project (3GPP). 3GPP is a worldwide organization for mobile wireless standards, which developed the first LTE standard (Release 8) in 2008. Several enhancements of the LTE standards have been published in subsequent releases since then.

As in a typical LTE network, the ground-based network infrastructure of the proposed AMT system will have base stations (also referred to as eNodeBs or eNBs) connected via backhaul links to the Enhanced Packet Core (EPC). The EPC includes the key elements of the LTE core network. An LTE User Equipment (UE), which is the LTE term for mobile devices that connect to the network, will be mounted on the TA to provide the communication link to carry telemetry data to the ground network. The UE will establish an LTE connection to the wireless infrastructure on the ground, which will then forward the telemetry data to the appropriate server. Fig. 2 shows an illustration of the proposed AMT network architecture.

As shown in Fig. 2, each eNB is connected to the Mobility Management Entity (MME) through an S1 interface on the control plane, and to the Serving GateWay (SGW) with an S1-U interface on the user plane. In LTE terms, the eNBs comprise the Radio Access Network (RAN), while the rest of the functional elements other than the Network Management System (NMS) and Integrated Cellular Network Controller (ICNC) comprise the LTE Evolved Packet Core (EPC). The RAN and the EPC together are referred to as the LTE network.

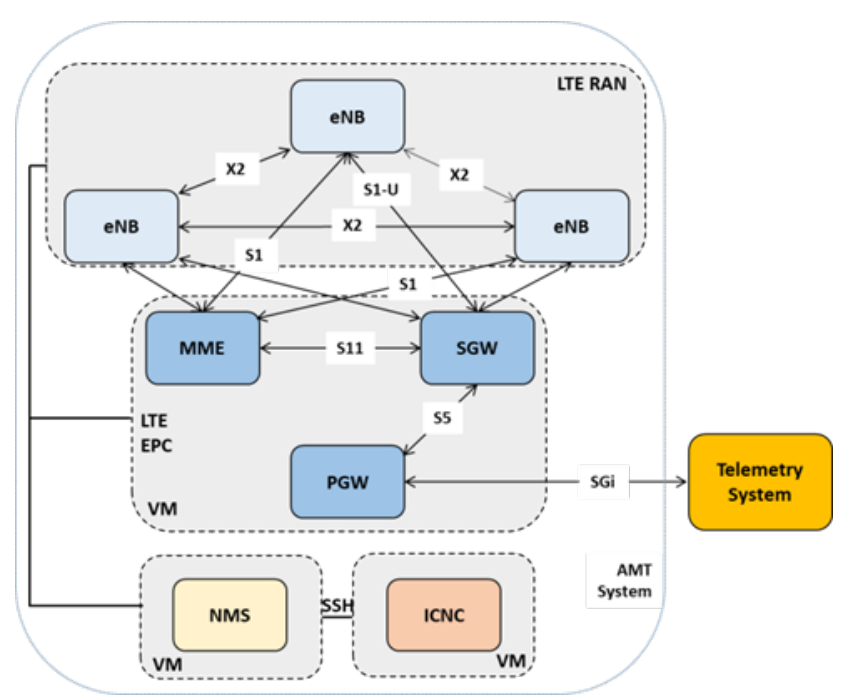

Fig. 2. An Illustration of the Network Architecture of the Proposed AMT System

It should be highlighted that the interfaces referred to in the previous paragraph are Internet Protocol (IP)-based and logical. That is, no direct eNB-eNB physical connection is required. The control plane's physical connection links can be routed through the MME if the MME is placed at a central location. The Evolved Packet Core delivers data to external elements, like the existing range telemetry system, via the SGi interface. The entire LTE network is managed through a Network Management System (NMS). Each vendor's implementation of the NMS is different as it is not a standardized element. However, the functionality remains the same; that is, all NMSs provide facilities to manage parameters and settings on all of the LTE network elements, and obtain key performance indicators (KPIs).

A key element of the proposed AMT system is the Integrated Cellular Network Controller (ICNC). The ICNC interacts with elements of the LTE network to force handovers to the desired eNB; the mechanism to do so is through the NMS. Parameters of the LTE network can be modified in real time through the interface to the NMS. The EPC, the NMS, and the ICNC can be located on the same server, with a virtualized implementation of each element.

Perspecta Labs installed a complete standalone LTE network at EAFB. The LTE RAN consisted of 3 LTE base stations (eNB1, eNB2 and eNB3) installed at different locations at EAFB. The objective was to provide coverage to the runway during taxiing and take-off and a broad open area while flying at low and high altitudes. The eNBs operated with power amplifiers and 4-way receive diversity in the Lower C-Band. The antennas had a broad beamwidth in azimuth and elevation and were up-tilted for maximum elevation-angle-range coverage. The 
three eNBs' backhaul connections to the LTE core network were wireline, providing IP connectivity. The ICNC was communicating in real time with the eNBs receiving real-time reports from the physical and RRC layers on the status of the connections.

Perspecta Labs' Velocite airborne LTE transceiver consisted of a COTS LTE terminal, an RF power amplifier and a Doppler Compensation appliqué based on software-defined radio. The system was installed on a rack and mounted in the pressurized cabin of a Beechcraft C$12 \mathrm{~J}$ aircraft. Two antennas, both situated at the aft and bottom of the fuselage were used for the test. The aircraft flew at different altitudes up to $20,000 \mathrm{ft}$ and at up to 253KGS (knots Ground Speed) during the test. The Velocite transceiver, would connect to a File Transfer Protocol (FTP) server on the ground through the overthe-air LTE connection. The FTP server was connected to the SGi interface of the LTE PGW node. The airborne Velocite transceiver would connect to the FTP server and generate IP traffic in both directions. A layer was built on top of this iperf application to enable optimized operation in the presence of disruptions in the radio link whose performance. The link was assessed through the iperf-provided statistics.

Actual time and position information was recorded during the flight, and compared with the LTE system measurements after the flight.

\section{Flight Tests: Results and Analysis}

The flight tests were carried out over two days October 30-31, 2019. On each day, a specific flight plan was prepared and executed. Each flight plan comprised a take-off segment and various routes including linear segments and circular paths. These routes tested different features of the LTE-based AMT system. Figure 3 shown below illustrates the flight plan executed on the first day. The figure also shows the three base stations (with their respective orientations) that constituted the ground segment of the AMT system. A similarly complex flight plan was executed during the second day of flight tests.

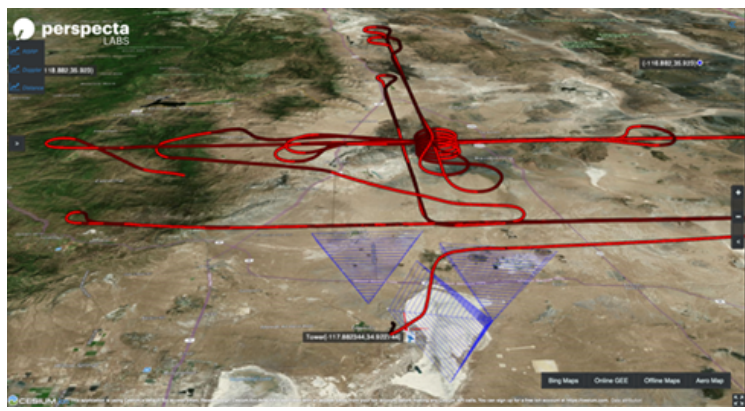

Fig. 3. Flight Plan Executed on the First Day
During the flight tests, a large amount of data was collected. While the main focus of the test was to study the system's ability to maintain the bi-directional link between the airborne LTE terminal and the ground-based base stations and deliver high throughput, data on a large number of parameters was collected to study how they affected system performance. The more important among them were the Doppler shift, distance, speed, received signal strength (RSRP), handover successes and failures.

On-board systems provided Time-SpacePosition Information (TSPI) data, which included the GPS-synchronized universal time, instantaneous location (latitude, longitude, altitude), speed, and heading of the TA. This data was collected in real-time. The Doppler shift, distance from each eNB, and the Line-Of-Sight (LOS) angle of arrival of RF signals were then calculated from the TSPI data after the test was complete. The Doppler shift calculated in this manner is referred to as the actual Doppler.

During the test, the CeRTN ICNC software was used to capture LTE protocol messages generated by the eNB. These were signaling messages associated with the link between the LTE terminal and the eNB that provided information regarding the reported $\mathrm{RSRP}$, timing advance, connectivity, and pending handovers. They also included information on any neighboring eNBs that were detected by the terminal. The information on neighbor eNBs was limited to RSRP, eNB ID, and pending handovers.

Figures $4 \mathrm{a}-4 \mathrm{c}$, shown below, are illustrative of some of the data collected during the tests. Fig. $4 a$ shows the RSRP from the serving and neighbor base stations as reported by the LTE terminal. Successful handover events (represented by green vertical lines) have been superimposed on the RSRP measurements. Note that RSRP measurements from neighbor base stations are available only if it is strong enough to be detected by the LTE terminal. Notice the close bunching of handover events near the 09:33 mark. Here, the RSRP levels associated with two or three base stations are close to each other. As a result, relatively small fluctuations in the measured RSPP levels cause corresponding changes in the base station seen by the terminal as the best to connect to, leading to frequent handovers. It is interesting to note that the distances from the base stations involved in the handovers to the LTE terminal are close to each other during this phase. (See fig. 4b.) Fig. 4c shows the actual and estimated Doppler shift for eNB1 and eNB2 during the take-off phase. (The estimated Doppler is referred to as measured Doppler in the figure.) Note that the appliqué estimates Doppler only 
for the serving base station. It is clear that the measured (estimated) Doppler closely tracks the actual Doppler for the serving base station regardless of the base station it is connected to.

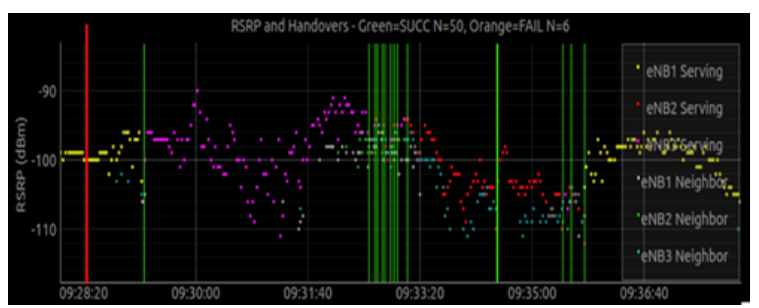

Fig. 4a. RSRP and Handovers during Takeoff Phase

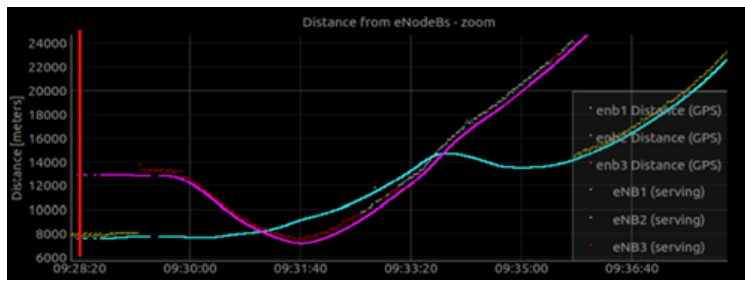

Fig. 4b. Distance to Base Stations during Takeoff Phase

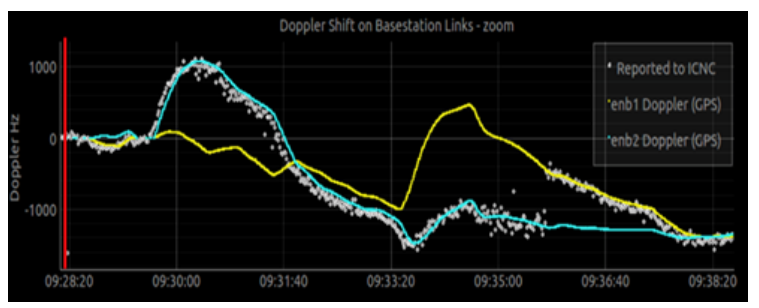

Fig. 4c. Doppler Estimation during Takeoff Phase

One of the important characteristics that was sought to be tested was the maximum range for the wireless connection between the LTE terminal and its serving base station. In order to determine the maximum range, the fight path included a segment where the TA was flown in a straight line until the connection was dropped.

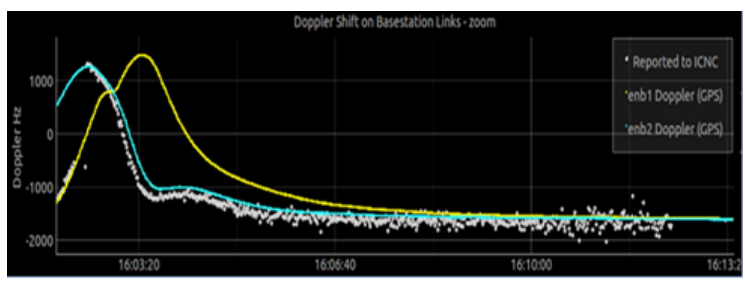

Fig. 5a. Doppler Shifts during Max. Range Phase

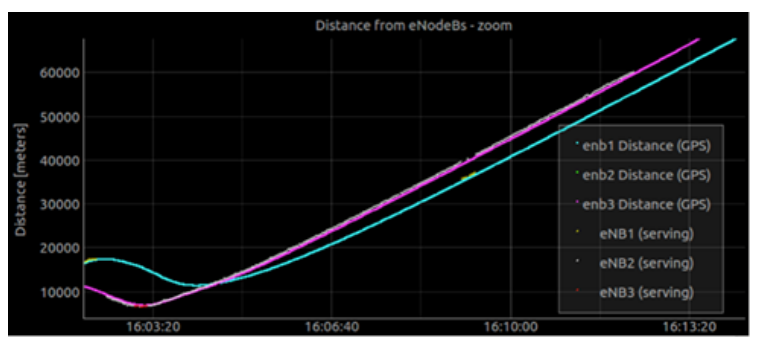

Fig. 5b. Distances to Base Stations during Maximum Range Phase
Figures $5 \mathrm{a}$ and $5 \mathrm{~b}$ respectively show the measured and actual Doppler shifts and the distances to the base stations eNB1 and eNB2 during this segment. As one can see from these figures, the flight test determined the maximum range for the LTE connection to be around 60 $\mathrm{km}$. Also, it is evident that the appliqué was able to accurately estimate and compensate for the Doppler shift over this entire range.

Data throughputs for the downlink and uplink directions during the maximum-range segment are shown in figs. $6 a$ and $6 \mathrm{~b}$, respectively. They show clear correlation to distance dependent pathloss with strong peak data rate near the eNBs and a sustained data rate up to $60 \mathrm{Km}$.

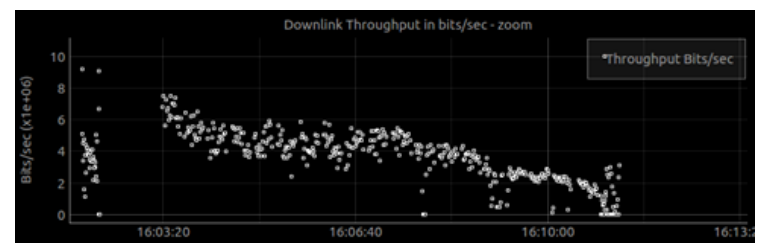

Fig. 6a. Ground-to-Air (DL) Data Throughput

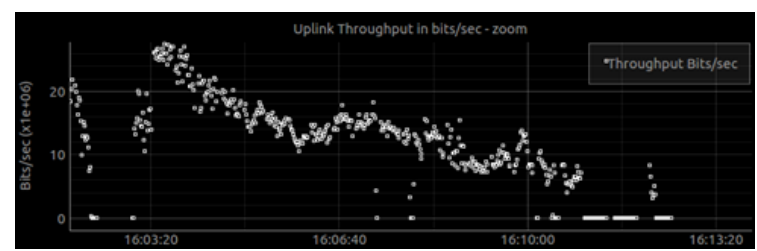

Fig. 6b. Air-to-Ground (UL) Data Throughput

We now present plots of the probability distribution functions (PDFs) of uplink (UL, i.e. air-toground) and downlink (DL, i.e. ground-to-air) throughputs and the Doppler shifts that were observed during the course of the tests.

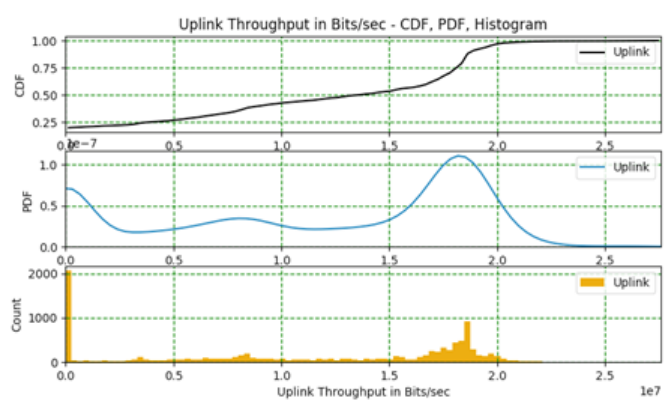

Fig. 7a. PDF of Uplink Throughput

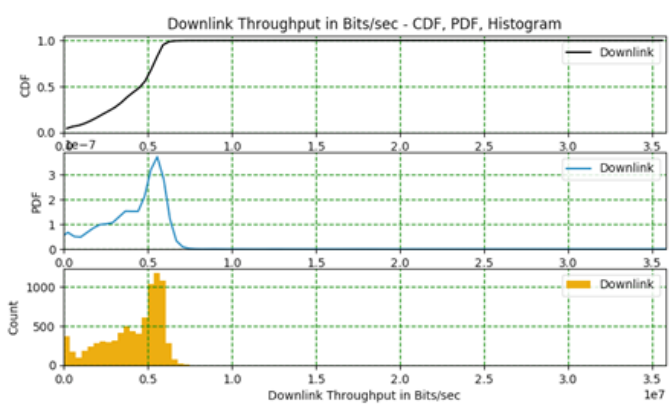

Fig. 7b. PDF of Downlink Throughput 
Figures $7 \mathrm{a}, 7 \mathrm{~b}$ respectively show the PDFs of UL and DL throughputs. As these figures show, the median UL throughput was 13 Mbps with the $75^{\text {th }}$ percentile at $18 \mathrm{Mbps}$, while the median $\mathrm{DL}$ throughput was $4.5 \mathrm{Mbps}$. The disparity between the UL and DL throughputs was due to the fact that in the Time-Division-Duplex (TDD) scheme used in the wireless link, the UL connection had access to the wireless medium $80 \%$ of the time. The probability mass at zero can be attributed mainly to the following causes: some quirks in the iperf software used to generate traffic, and breaks in the link during certain maneuvers that caused the TA antenna to be obscured by the aircraft body.

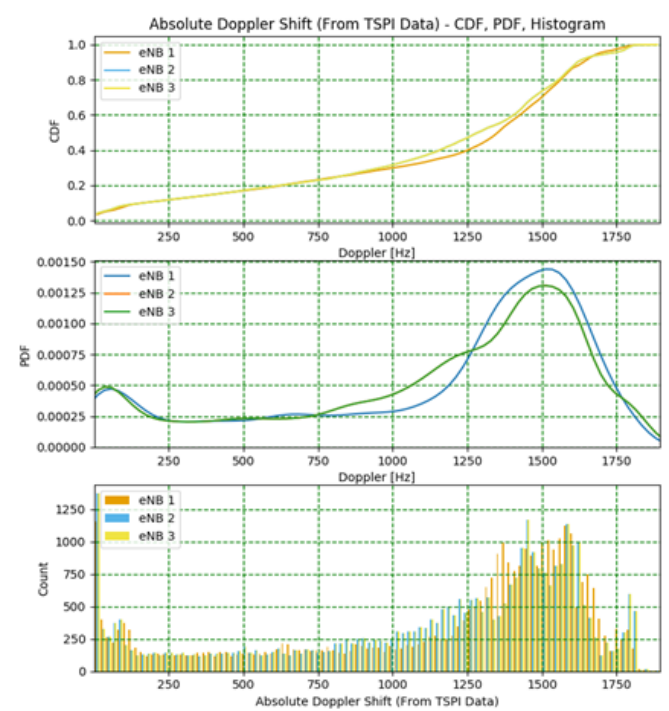

Fig. 8. PDF of Doppler Shifts

Fig. 8 shows the PDF of the Doppler shifts observed during the flight tests. As can be seen in the figure, most of the probability mass in the Doppler shift associated with both base stations is located between 1.20 and $1.75 \mathrm{kHz}$, which is well beyond the tolerance of COTS base station receivers. However, as demonstrated throughout this test, the combination of the LTE modem with the Doppler compensation appliqué enables the AMT system to maintain a goodquality link to the base stations on the ground, delivering a high throughput.

We conclude this section with some remarks on the handovers observed during the flight tests. Handovers enable seamless connectivity in the cellular-based AMT system, and a high rate of handover successes indicate a well-designed network. During the flight test, a large number of handovers were observed (more than 140) with an overall success rate of $82 \%$. This demonstrated the agility of the prototype system to follow the LTE signaling procedures. Moreover, the data showed us areas where the handover thresholds and hysteresis levels needed further fine-tuning to adjust to the flight characteristics.

\section{Conclusions}

The flight tests carried out at EAFB to study the viability of the Velocite AMT system demonstrated a strong agility to adapt to varying high Doppler shifts. The prototype system used in the test showed that it was able to estimate and compensate for the Doppler shift at the serving base station under a variety of challenging conditions, helping to maintain link connectivity and delivering a high throughput. It was also demonstrated that the system could support a maximum range of $60 \mathrm{~km}$ and a high probability of handover success. Overall, it was shown that the addition of a Doppler appliqué on a COTS LTE modem at the airborne system in combination with a well-designed ground network enables a full duplex AMT link over large areas that can be covered with relatively few cells equipped with broad-beam antennas. Future prototypes are expected to provide enhancements for higher speeds and more features to further improve the robustness of the radio link.

\section{Acknowledgements}

This work is being funded by NSC Project No.: W15QKN-15-9-1004 for the T\&E S\&T SET Program at Edwards Air Force Base.

\section{References}

[1] A. Kogiantis, K. Rege and A. Triolo, "LTE System Architecture for Coverage and Doppler Reduction in Range Telemetry," presented at The International Telemetering Conference, Las Vegas, Nevada, Oct. 23-26, 2017.

[2] E. Fung, W.H. Johnson, A. Kogiantis and K.M. Rege, "Doppler Estimation and Compensation for LTE-Based Aeronautical Mobile Telemetry," presented at The International Telemetering Conference, Glendale, Arizona, Nov. 5-8, 2018.

[3] S. Sesia, I. Toufik, and M. Baker (eds.), LTE The UMTS Long Term Evolution - From Theory to Practice, Second Edition, John Wiley and Sons, Chichester, U.K., 2011. 